\title{
Prevalence and reactivity of anti-melanoma differentiation-associated gene 5 (anti-MDA-5) autoantibody in Brazilian patients with dermatomyositis ${ }^{*}$
}

\author{
Isabela Bruna Pires Borges ${ }^{1}$, Marilda Guimarães Silva ${ }^{1}$, Samuel Katsuyuki Shinjo ${ }^{1}$
}

DOI: http:/ / dx.doi.org/10.1590/abd1806-4841.20186803

\begin{abstract}
BACKGROUND: There have been no studies to date on the frequency and reactivity of aanti-melanoma differentiation-associated gene 5 (anti-MDA-5) in samples from the Brazilian population with dermatomyositis.

OвJестіvEs. To analyze this autoantibody in the Brazilian population.

Methods: This was a single-center cross-sectional study in which 131 consecutive adult patients (109 dermatomyositis and 22 clinically amyopathic dermatomyositis) with active disease were evaluated from 2000 to 2016. Analysis of the anti-MDA-5 autoantibody was performed by ELISA.

RESULTS: The presence of this autoantibody was observed in $14.7 \%$ and $22.7 \%$ of patients with dermatomyositis and clinically amyopathic dermatomyositis, respectively. In the case of dermatomyositis, the autoantibody was associated less frequently with Raynaud's phenomenon and periungual hyperemia $(\mathrm{P}<0.05)$. In clinically amyopathic dermatomyositis, the presence of this autoantibody was not associated statistically with any demographic, clinical, laboratory, or imaging characteristics.

STUDY LIMITATIONS: The cross-sectional study design did not allow establishing a temporal correlation between anti-MDA-5 autoantibody and various study variables. In addition, pulmonary function tests were not performed in the patients.

CONCLUSIONS. The frequency of anti-MDA-5 autoantibody was comparable to that of other populations with dermatomyositis, but with a different reactivity than described in the literature. In addition, there was a phenotypic variability between our patients with clinically amyopathic dermatomyositis and those described in the literature. Further studies are needed to confirm the current study's findings and elucidate this autoantibody's reactivity in Brazilians with idiopathic inflammatory myopathies.
\end{abstract}

Keywords: Antibodies; Cross-sectional studies; Dermatomyositis; Epidemiological study characteristics; Myositis

\section{INTRODUCTION}

Dermatomyositis (DM) is an autoimmune inflammatory myopathy characterized by muscle weakness of the limbs, mainly proximal, symmetric, and progressive, in addition to classic cutaneous alterations such as heliotrope rash and Gottron's papules. It can also present other skin lesions such as cuticular hypertrophy, periungual hyperemia, photosensitivity, calcinosis, "V-neck sign", and "shawl sign", among others. ${ }^{1-3}$

In $10-15 \%$ of cases, DM patients do not present muscle involvement, thus characterizing the DM subset known as amyopathic or sine myositis., ${ }^{4,5} \mathrm{DM}$ can also be classified as hypomyopathic, with altered laboratory results, evidencing some degree of myopathy. These two forms of DM (amyopathic and hypomyopathic) are called clinically amyopathic DM..$^{2,5}$

Sato et al. ${ }^{6}$ identified the autoantibody to the $140-\mathrm{kDa}$ clinically amyopathic dermatomyositis peptide (CADM-140) in Japanese patients with clinically amyopathic DM and whose principal characteristic was rapidly progressive pulmonary involvement. The target antigen for this autoantibody is the 140 kDa cytoplasmic protein called melanoma differentiation associated gene 5 (MDA-5), an RNA-specific helicase. ${ }^{7}$ Therefore, antiCADM-140 is now also referred to as anti-MDA-5. ${ }^{7}$

Received 20 December 2016.

Accepted 23 June 2017.

* Work conducted at the Division of Rheumatology, Faculdade de Medicina da Universidade de São Paulo, São Paulo (SP), Brazil.

Financial support: FAPESP \#2015/12628-0 (IBPB) and \#2014/09079-1 (SKS); CAPES (MGS).

Conflict of interest: None.

1 Division of Rheumatology, Faculdade de Medicina, Universidade de São Paulo, São Paulo (SP), Brasil.

MaILING AdDREss:

Samuel Katsuyuki Shinjo

E-mail: samuel.shinjo@gmail.com

(C)2018 by Anais Brasileiros de Dermatologia 
The clinical characteristics and prognosis of patients with anti-MDA-5 autoantibodies are variable. The literature mentions the relationship between anti-MDA- 5 and skin eruptions, rapid and progressive development of interstitial lung disease, skin lesions (rash, palmar papules, and/or ulcerations), joint and lung involvement, and/or worse prognosis in DM patients. ${ }^{7-13}$ Some of these patients also presented a similar phenotype to that of anti-synthetase syndrome.$^{14}$ However, this wide phenotypical variability between the studies on the association with anti-MDA- 5 autoantibody may be due to the heterogeneity of the populations studied, thus justifying the importance of assessing Brazilian patients with idiopathic inflammatory myopathies.

These studies also failed to mention possible confounders (smoking, chronic obstructive pulmonary disease, and overlapping of other systemic autoimmune diseases such as systemic sclerosis, systemic lupus erythematosus, and Sjögren's syndrome, among others), which could interfere in the correlation between anti-MDA-5 autoantibody and the patients' clinical and laboratory parameters. ${ }^{8,10-16}$

Presence of anti-MDA-5 autoantibody had not been analyzed previously in Brazilian patients with DM, thus justifying the current study, with the aim of verifying the autoantibody's prevalence in patients with DM and clinically amyopathic DM, without possible confounders. The study also aimed to assess a possible association between the autoantibody and demographic, clinical, laboratory, and imaging parameters.

\section{METHODS}

This was a single-center cross-sectional study to evaluate the presence of anti-MDA- 5 autoantibody in adult patients with $\mathrm{DM}$, defined according to the criteria proposed by Bohan and Peter. ${ }^{1}$ Clinically amyopathic DM was determined according to the criteria of Gerami et al. ${ }^{16}$ All the assessed patients came from the same tertiary service, from 2000 to 2016.

The study was approved by the local Institutional Review Board (number 1.483.411).

The exclusion criteria were: patients with overlapping systemic autoimmune diseases, neoplasms, pulmonary infections (tuberculosis, aspergilloma), or chronic obstructive pulmonary disease; history of smoking; history of prior exposure to statins or fibrates; and suspected cases of muscular dystrophies.

Of the 218 patients that were initially admitted, 87 were excluded after application of the exclusion criteria. We thus analyzed 131 consecutive cases, of which 109 (83.2\%) were DM and 22 (16.8\%) clinically amyopathic DM.

The following data from the eligible patients were assessed, based on the electronic patient files, with pre-standardized and parameterized information, covering the data from the initial diagnostic workup and follow-up of patients with clinical and laboratory activity:

1. Demographics: current age; age at diagnosis of the disease; time between diagnosis and onset of symptoms; sex and race/color;

2. Clinical manifestations: constitutional symptoms (fever and weight loss); heliotrope rash; Gottron's sign/papules; facial rash; "V-neck sign"; "shawl sign"; periungual hyperemia; vasculitis; calcinosis; ulcers; Raynaud's phenomenon; mechanic's hands; muscle weakness in the upper and lower limbs; Medical Research Council muscle strength grading; joint, gastrointestinal (high dysphagia), and pulmonary involvement (dyspnea: on moderate to small efforts; rapidly evolving dyspnea: in less than three months since onset of general symptoms); ${ }^{17}$

3. Altered pulmonary images obtained by CT: incipient lung disease, "ground glass" opacity, and bilateral basal pulmonary fibrosis;

4. Serum muscle enzyme levels in routine blood samples taken for medical consultation: creatinine phosphokinase (reference value: 32 - 294U/L) and aldolase (1.0 - 7.5U/L), performed with automated kinetic method.

Analysis of anti-MDA-5 autoantibody used serum samples in aliquots previously stored at $-20^{\circ} \mathrm{C}$, collected during the initial workup in patients with clinical and laboratory activity. The autoantibody was identified with enzyme-linked immunosorbent assay (ELISA), through the MDA-5 recombinant protein and anti-MDA-5 monoclonal antibody (MyBioSource, CA, USA). For the purposes of this study and to ensure positive results for anti-MDA-5, positivity was defined as patients with values more than three standard deviations above the mean in eight controls.

\section{Statistical analysis}

The Kolmogorov-Smirnov test was used to assess the distribution of each of the continuous variables. The results were presented as mean \pm standard deviation for continuous variables and number (\%) for categorical variables. Median values (interquartile range, $25 \%-75 \%$ ) were calculated for continuous variables that did not display normal distribution. Data on presence versus absence of anti-MDA-5 autoantibody were compared with Students t-test or Mann-Whitney test for continuous variables. Differences in categorical variables were calculated with Pearsons $\mathrm{c}^{2}$ or Fisher's exact test. Statistical significance was set at $p<0.05$.

\section{RESULTS}

Of the 131 patients, $108(83.2 \%)$ and $22(16.8 \%)$ presented $\mathrm{DM}$ and clinically amyopathic DM, respectively. Table 1 shows these patients' demographic, clinical, laboratory, and imaging data Age at diagnosis was 41.3 and 49.7 years, respectively, in patients with DM and clinically amyopathic DM. Female gender and white color predominated in both groups.

Constitutional symptoms were present in more than half of the cases in both groups (Table 1).

The principal cutaneous manifestations were heliotrope rash and Gottron's sign/papules, followed by secondary lesions (facial rash, "V-neck" sign, "shawl" sign, periungual hyperemia, vasculitis, calcinosis, mechanic's hand, ulcers, and Raynaud's phenomenon).

As expected, muscle weakness in the limbs and elevated serum muscle enzymes were more evident in the classic form of DM, compared to clinically amyopathic DM.

Joint involvement and dysphagia were also found in both groups.

Dyspnea was reported in $30.3 \%$ and $31.8 \%$ of patients with DM and clinically amyopathic DM, respectively, while rapidly evolving dyspnea was present in $13.8 \%$ and $4.6 \%$ of the patients. In 
TABLE 1: Demographic, clinical, laboratory, and imaging data of patients with dermatomyositis (classic and clinically amyopathic forms)

\begin{tabular}{|c|c|c|c|}
\hline & DM (N=109) & CADM (N=22) & TOTAL $(\mathrm{N}=131)$ \\
\hline Age at diagnosis (years) & $41.3 \pm 14.4$ & $49.7 \pm 14.7$ & $41.3 \pm 14.4$ \\
\hline Time between diagnosis and symptoms (months) & $4(2-7)$ & $4(2-12)$ & $6(0-36)$ \\
\hline Female & $84(77.1)$ & $12(54.6)$ & $96(73.3)$ \\
\hline White & $91(83.5)$ & $18(81.8)$ & $109(83.2)$ \\
\hline Constitutional symptoms & $69(63.3)$ & $12(54.6)$ & $81(61.8)$ \\
\hline \multicolumn{4}{|l|}{ Cutaneous } \\
\hline Heliotrope rash & $92(84.4)$ & $19(86.4)$ & $111(84.7)$ \\
\hline Gottron's sign/papules & $98(89.9)$ & $21(95.5)$ & $119(90.8)$ \\
\hline Facial rash & $69(63.3)$ & $16(72.7)$ & $85(64.9)$ \\
\hline "V-neck" sign & $47(43.1)$ & $10(45.5)$ & $57(43.5)$ \\
\hline "Shawl" sign & $30(27.5)$ & $6(27.3)$ & $36(27.5)$ \\
\hline Periungual hyperemia & $67(61.5)$ & 17 (77.3) & $84(64.1)$ \\
\hline Vasculitis & $26(23.9)$ & $8(36.4)$ & $34(26.0)$ \\
\hline Calcinosis & $10(9.2)$ & $2(9.1)$ & $12(9.2)$ \\
\hline Mechanic's hands & $22(20.2)$ & $3(13.6)$ & $25(19.1)$ \\
\hline Ulcers & 19 (17.4) & $5(22.7)$ & $24(18.3)$ \\
\hline Raynaud's phenomenon & $52(47.7)$ & $13(59.1)$ & $65(49.6)$ \\
\hline \multicolumn{4}{|l|}{ Muscle weakness } \\
\hline \multicolumn{4}{|l|}{ Upper limbs } \\
\hline Grade V & $1(0.9)$ & $15(68.2)$ & $16(12.2)$ \\
\hline Grade IV & $69(63.3)$ & $7(31.8)$ & $76(58.0)$ \\
\hline Grade III & $39(35.8)$ & 0 & $39(29.8)$ \\
\hline Grade II & 0 & 0 & 0 \\
\hline Grade I & 0 & 0 & 0 \\
\hline \multicolumn{4}{|l|}{ Lower limbs } \\
\hline Grade V & $2(1.8)$ & $14(63.6)$ & $16(12.2)$ \\
\hline Grade IV & $74(67.9)$ & $8(36.4)$ & $82(62.6)$ \\
\hline Grade III & $31(28.4)$ & 0 & $31(23.7)$ \\
\hline Grade II & $2(1.8)$ & 0 & $2(1.5)$ \\
\hline Grade I & 0 & 0 & 0 \\
\hline Joint & $31(28.4)$ & $9(40.9)$ & $40(30.5)$ \\
\hline Gastrointestinal (dysphagia) & $59(54.1)$ & $5(22.7)$ & $64(48.9)$ \\
\hline \multicolumn{4}{|l|}{ Pulmonary } \\
\hline Dyspnea & $33(30.3)$ & $7(31.8)$ & $40(30.5)$ \\
\hline Dyspnea (<3 months) & $15(13.8)$ & $1(4.6)$ & $16(12.2)$ \\
\hline \multicolumn{4}{|l|}{ Chest CT } \\
\hline "Ground glass" & $15(13.8)$ & $5(22.7)$ & $20(15.3)$ \\
\hline Incipient lung disease & $33(30.3)$ & $7(31.8)$ & $40(30.5)$ \\
\hline Pulmonary fibrosis & $8(7.3)$ & $1(4.6)$ & $9(6.9)$ \\
\hline Creatine phosphokinase (U/L) & $1831(29-8000)$ & $110(124-170)$ & $4435(28-22858)$ \\
\hline Aldolase (U/L) & $24.7(8.3-60.8)$ & $9.7(5.5-15.3)$ & $51.2(2.9-415.5)$ \\
\hline Anti-MDA-5 & $16(14.7)$ & $5(22.7)$ & $21(16.0)$ \\
\hline
\end{tabular}

CADM: Clinically amyopathic dermatomyositis; MDA: melanoma differentiation-associated gene 5.

Data expressed as mean \pm standard deviation, median (interquartile range $25 \%-75 \%$ ), or percentage (\%). 
addition, the main CT finding was presence of incipient lung disease in both groups (one third of cases).

The anti-MDA-5 autoantibody was present in $14.7 \%$ of patients with DM and $22.7 \%$ of these with clinically amyopathic DM (Table 1).

We also compared patients with DM (classic form) according to presence or absence of anti-MDA-5 autoantibody [MDA-5(+) vs. MDA-5(-)] (Table 2). Age, gender, race/color, and time between diagnosis and onset of simptoms were similar in the two groups. The presence of constitutional symptoms, clinical manifestations, and laboratory and imaging results were also comparable between the two groups, except for the lower frequency of periungual hyperemia $(25 \%$ vs. $67.7 \%$; $\mathrm{p}<0.002)$ and Raynaud's phenomenon $(18.8 \%$ vs. $52.7 \%$; $\mathrm{p}<0.015)$ in anti-MDA-5(+) compared to anti-MDA-5 (-) patients.

In patients with clinically amyopathic DM, all the demographic, clinical, laboratory, and imaging parameters were similar in those with MDA-5(+) and MDA-5(-) (Table 3).

TABLE 2: Demographic, clinical, laboratory, and imaging data from patients with dermatomyositis (classic from) in relation to anti-MDA-5 autoantibody positivity

\begin{tabular}{|c|c|c|c|}
\hline & MDA-5 (+) $(\mathrm{N}=16)$ & MDA-5 (-) (N=93) & p-value \\
\hline Age at diagnosis (years) & $41.8 \pm 14.0$ & $40.5 \pm 14.5$ & 0.749 \\
\hline Time between diagnosis and symptoms (months) & $3(1-5)$ & $4(3-7)$ & 0.067 \\
\hline Female & $13(81.2)$ & $71(76.3)$ & 1.000 \\
\hline White & $15(93.8)$ & $76(81.7)$ & 1.000 \\
\hline Constitutional symptoms & $11(68.8)$ & $58(62.4)$ & 0.782 \\
\hline \multicolumn{4}{|l|}{ Cutaneous } \\
\hline Heliotrope rash & $13(81.3)$ & $79(85.0)$ & 0.713 \\
\hline Gottron's sign/papules & $14(87.5)$ & $84(90.3)$ & 0.663 \\
\hline Facial rash & $11(68.8)$ & $58(62.4)$ & 0.781 \\
\hline "V-neck" sign & $5(31.3)$ & $42(45.2)$ & 0.414 \\
\hline "Shawl" sign & $1(6.3)$ & $29(31.2)$ & 0.065 \\
\hline Periungual hyperemia & $4(25.0)$ & $63(67.7)$ & 0.002 \\
\hline Vasculitis & $4(25.0)$ & $22(23.7)$ & 1.000 \\
\hline Calcinosis & $1(6.3)$ & $9(9.7)$ & 1.000 \\
\hline Mechanic's hands & $1(6.3)$ & $21(22.6)$ & 0.185 \\
\hline Ulcers & $4(25.0)$ & $15(16.1)$ & 0.474 \\
\hline Raynaud's phenomenon & $3(18.8)$ & $49(52.7)$ & 0.015 \\
\hline \multicolumn{4}{|l|}{ Muscle weakness } \\
\hline \multicolumn{4}{|l|}{ Upper limbs } \\
\hline Grade V & 0 & $1(1.1)$ & \\
\hline Grade IV & $3(18.8)$ & $66(71.0)$ & \\
\hline Grade III & $13(81.2)$ & $26(27.9)$ & 0.827 \\
\hline Grade II & 0 & 0 & \\
\hline Grade I & 0 & 0 & \\
\hline \multicolumn{4}{|l|}{ Lower limbs } \\
\hline Grade V & $1(6.3)$ & $1(1.1)$ & \\
\hline Grade IV & $10(62.4)$ & $64(68.7)$ & \\
\hline Grade III & $5(31.3)$ & $26(28.0)$ & 0.733 \\
\hline Grade II & 0 & $2(2.2)$ & \\
\hline Grade I & 0 & 0 & \\
\hline Join & $5(31.3)$ & $26(28.0)$ & 0.771 \\
\hline Gastrointestinal (dysphagia) & $6(37.5)$ & $53(57.0)$ & 0.180 \\
\hline \multicolumn{4}{|l|}{ Pulmonary } \\
\hline Dyspnea & $4(25.0)$ & $29(31.2)$ & 0.772 \\
\hline Dyspnea (<3 months) & $2(12.5)$ & $13(14.0)$ & 1.000 \\
\hline \multicolumn{4}{|l|}{ Chest CT } \\
\hline “Ground glass" & $1(6.3)$ & $14(15.1)$ & 0.693 \\
\hline Incipient lung disease & $4(25.0)$ & $29(31.2)$ & 0.772 \\
\hline Pulmonary fibrosis & 0 & $8(8.6)$ & - \\
\hline Creatine Phosphokinase (U/L) & $3726(440-5884)$ & $1341(340-8067)$ & 0.783 \\
\hline Aldolase (U/L) & $39.6(6.3-60.8)$ & $23.4(8.4-68.9)$ & 0.975 \\
\hline
\end{tabular}

MDA: melanoma differentiation-associated gene 5 .

Data expressed as mean \pm standard deviation, median (interquartile range $25 \%-75 \%$ ), or percentage ( $\%$ ). 


\section{DISCUSSION}

The study showed that the frequency of anti-MDA-5 autoantibody in Brazilian patients with DM and clinically amyopathic DM was consistent with that reported in other populations, but with different reactivity from that described in the literature.

The study's strengths include the evaluation of presence of anti-MDA-5 autoantibody in patients with a well-defined diagnosis of DM, based on the criteria proposed by Bohan and Peter ${ }^{1}$ and thus excluding cases with probable or possible diagnoses. For cases of clinically amyopathic DM, we used the updated definition proposed by Gerami et al. ${ }^{5}$ In addition, unlike previous studies in the literature, we applied rigorous exclusion criteria, for example patients with history of lung disease, tuberculosis, other systemic autoimmune diseases, neoplasms, smoking, and others, since such diseases, comorbidities, or habits could act as confounding factors for the interpretation and association between presence of anti-MDA-5 autoantibody and the clinical presentation of patients with DM or clinically amyopathic DM.

TABLE 3: Demographic, clinical, laboratory, and imaging data from patients with clinically amyopathic dermatomyositis in relation to anti-MDA-5 autoantibody positivity

\begin{tabular}{|c|c|c|c|}
\hline & MDA-5 (+) $(\mathrm{N}=5)$ & MDA-5 (-)(N=17) & Valor de $p$ \\
\hline Age at diagnosis (years) & $44.2 \pm 15.5$ & $44.0 \pm 13.5$ & 0.981 \\
\hline Time between diagnosis and symptoms (months) & $3(1-8)$ & $5(3-18)$ & 0.359 \\
\hline Female & $2(40.0)$ & $10(58.8)$ & 0.624 \\
\hline White & $4(80.0)$ & $14(82.4)$ & 1.000 \\
\hline Constitutional symptoms & $3(60.0)$ & $9(52.9)$ & 1.000 \\
\hline \multicolumn{4}{|l|}{ Cutaneous } \\
\hline Heliotrope rash & $4(80.0)$ & $15(88.2)$ & 1.000 \\
\hline Gottron's sign/papules & $5(100.0)$ & $16(94.1)$ & 0.455 \\
\hline Facial rash & $3(60.0)$ & $13(76.5)$ & 0.585 \\
\hline "V-neck" sign & 0 & $10(58.8)$ & - \\
\hline "Shawl" sign & 0 & $6(35.3)$ & - \\
\hline Periungual hyperemia & $5(100)$ & $12(70.6)$ & 0.290 \\
\hline Vasculitis & $2(40.0)$ & $6(35.3)$ & 1.000 \\
\hline Calcinosis & 0 & $2(11.8)$ & - \\
\hline Mechanic's hands & $1(20.0)$ & $2(11.8)$ & 1.000 \\
\hline Ulcers & $1(20.0)$ & $4(23.5)$ & 1.000 \\
\hline Raynaud's phenomenon & $2(40.0)$ & $11(64.7)$ & 0.609 \\
\hline \multicolumn{4}{|l|}{ Muscle weakness } \\
\hline \multicolumn{4}{|l|}{ Upper limbs } \\
\hline Grade V & $2(40.0)$ & $13(76.5)$ & \\
\hline Grade IV & $3(60.0)$ & $4(23.5)$ & \\
\hline Grade III & 0 & 0 & 0.249 \\
\hline Grade II & 0 & 0 & \\
\hline Grade I & 0 & 0 & \\
\hline \multicolumn{4}{|l|}{ Lower limbs } \\
\hline Grade V & $3(60.0)$ & $11(64.7)$ & \\
\hline Grade IV & $2(40.0)$ & $6(35.3)$ & \\
\hline Grade III & 0 & 0 & 0.880 \\
\hline Grade II & 0 & 0 & \\
\hline Grade I & 0 & 0 & \\
\hline Joint & $2(40.0)$ & $7(41.2)$ & 1.000 \\
\hline Gastrointestinal (dysphagia) & $1(20.0)$ & $4(23.5)$ & 1.000 \\
\hline \multicolumn{4}{|l|}{ Pulmonary } \\
\hline Dyspnea & $1(20.0)$ & $6(35.3)$ & 1.000 \\
\hline Dyspnea (<3 months) & 0 & $1(5.9)$ & - \\
\hline \multicolumn{4}{|l|}{ Chest CT } \\
\hline “Ground glass" & $1(20.0)$ & $4(23.5)$ & 1.000 \\
\hline Incipient lung disease & $2(40.0)$ & $5(29.4)$ & 1.000 \\
\hline Pulmonary fibrosis & 0 & $1(5.9)$ & - \\
\hline Creatine phosphokinase (U/L) & $167(103-527)$ & $105(58-159)$ & 0.330 \\
\hline Aldolase (U/L) & $15.2(11.2-22.7)$ & $7.8(4.0-10.5)$ & 0.067 \\
\hline
\end{tabular}

MDA: melanoma differentiation-associated gene 5.

Data expressed as mean \pm standard deviation, median (interquartile range $25 \%-75 \%$ ), or percentage ( $\%$ ). 
Although this was a retrospective study, the data had been previously standardized and parameterized and were thus trustworthy. In addition, in order to increase the specificity of the ELISA test, we applied a cutoff point of at least three standard deviations to include cases in the study, unlike previous studies such as Labrador-Horrillo et al., ${ }^{14}$ who used two standard deviations as their cutoff.

According to the literature, positivity for anti-MDA-5 autoantibody can vary from 4.7 to $13.1 \%$ in DM and 10.0 to $18.8 \%$ in patients with clinically amyopathic DM. ${ }^{7,11-14}$ In the current study, MDA-5 autoantibody was present in $14.7 \%$ of patients with DM and $22.7 \%$ in clinically amyopathic DM, thus corroborating the rates reported in the literature. Since anti-MDA-5 autoantibody can vary according to disease activity, it is important to note that the current study only included patients with clinical and laboratory activity. ${ }^{18-20}$

In patients with classic DM, anti-MDA-5 autoantibody was associated with lower frequency of Raynaud's phenomenon and periungual hyperemia. However, unlike other studies, we did not find an association between this autoantibody and the presence of skin ulcers, for example. ${ }^{12-14}$ Further studies are needed to better elucidate the relevance of these associations.

Neither did we observe an association between anti-MDA-5 autoantibody and a clinical presentation similar to anti-synthetase syndrome or its components (e.g., joint and pulmonary involvement). However, Hall et al. ${ }^{12}$ and Labrador-Horrillo et al. ${ }^{14}$ reported a high rate of anti-MDA-5 autoantibody in patients with symptoms suggestive of anti-synthetase syndrome, but without the presence of anti-synthetase autoantibody (e.g., anti-Jo-1 autoantibody). These discrepant data reinforce the presence of wide phenotypical variability among the different populations studied, as well as different anti-MDA-5 autoantibody reactivity according to the study population. Unlike our study, those authors did not mention possible confounding factors that could lead to lung or joint involvement in their series (e.g., overlapping diagnoses, history of smoking, chronic obstructive pulmonary disease (COPD), history of tuberculosis, etc.). ${ }^{12,14}$

In patients with clinically amyopathic DM we observed a variety of skin lesions, mainly Gottron's sign/papules, heliotrope rash, facial rash, and periungual hyperemia. These were distributed the same way in patients with or without presence of anti-MDA-5 autoantibody. In contrast, studies in the literature reported a strong association between this autoantibody and the skin lesions found in patients with clinically amyopathic DM. ${ }^{6,8,12-14}$

Pulmonary involvement in patients with clinically amyopathic DM is relatively common. ${ }^{6,20-24}$ Particularly in Japanese and Chinese populations, it has been reported frequently in patients with rapidly progressive interstitial lung disease. ${ }^{6,20-24}$ Meanwhile, the prevalence of rapidly progressive interstitial lung disease in clinically amyopathic DM is relatively low in the United States, as in the current study. ${ }^{25}$

Previous studies also showed a strong association between anti-MDA-5 autoantibody and presence of pulmonary manifestations, which we did not observe in this study. ${ }^{6,9,14}$ One explanation is that we found a low rate of patients with pulmonary involvement and rapidly progressive dyspnea. These findings in turn may be the consequence of phenotypical diversity in the study sample, as well as the use of strict exclusion criteria, especially for factors related to pulmonary involvement (COPD, smoking, etc.).

As limitations, the study used a cross-sectional design, which prevented establishing a temporal correlation between anti-MDA-5 autoantibody and various independent variables. In addition, lung function tests were not performed in the patients.

\section{CONCLUSIONS}

Anti-MDA-5 autoantibody was highly prevalent in patients with DM and clinically amyopathic DM, consistent with findings in other populations, but with different reactivity than described in the literature. The patients in our sample also had different clinical characteristics from those reported in the literature, i.e., without major pulmonary and/or cutaneous involvement and with no similarity to anti-synthetase syndrome.

The study's results can contribute to our understanding of different clinical, phenotypical, laboratory, and demographic characteristics of patients, thus allowing more individualized diagnosis and treatment. $\square$ 


\section{REFERENCES}

1. Bohan A, Peter JB. Polymyositis and dermatomyositis (first of two parts). N Engl J Med. 1975;292:344-7.

2. $\quad$ Dalakas MC. Inflammatory muscle diseases. N Engl J Med. 2015;372:1734-47.

3. Dalakas MC. Inflammatory muscle diseases. 2015;373:393-4.

4. Euwer RL, Sontheimer RD. Amyopathic dermatomyositis (dermatomyositis sine myositis). Presentation of six new cases and review of the literature. J Am Acad Dermatol. 1991;24:959-66.

5. Gerami P, Schope JM, McDonald L, Walling HW, Sontheimer RD. A systematic review of adult-onset clinically amyopathic dermatomyositis (dermatomyositis sine myositis): a missing link within the spectrum of the idiopathic inflammatory myopathies. J Am Acad Dermatol. 2006;54:597-613.

6. Sato S, Hirakata M, Kuwana M, Suwa A, Inada S, Mimori T, et al. Autoantibodies to a 140-kd polypeptide, CADM-140, in Japanese patients with clinically amyopathic dermatomyositis. Arthritis Rheum. 2005;52:1571-6.

7. Sato S, Hoshino K, Satoh T, Fujita T, Kawakami Y, Fujita T, et al. RNA helicase encoded by melanoma differentiation-associated gene 4 is a major autoantigen in patients with clinically amyopathic dermatomyositis: Association with rapidaly progressive interstitial lung disease. Arthritis Rheum. 2009;60:2193-200.

8. Narang NS, Casciola-Rosen L, Li S, Chung L, Fiorentino DF. Cutaneous ulceration in dermatomyositis: association with anti-melanoma differentiation-associated gene 5 antibodies and interstitial lung disease. Arthritis Care Res (Hoboken). 2015;67:667-72.

9. Hoshino K, Muro Y, Sugiura K, Tomita Y, Nakashima R, Mimori T. Anti-MDA5 and anti-TIF1-gamma antibodies have clinical significance for patients with dermatomyositis. Rheumatology (Oxford). 2010;49:1726-33.

10. Muro Y, Sugiura K, Hoshino K, Akiyama M, Tamakoshi K. Epidemiologic study of clinically amyopathic dermatomyositis and anti-melanoma differentiationassociated gene 5 antibodies in central Japan. Arthritis Res Ther. 2011;13:R214.

11. Moghadam-Kia S, Oddis CV, Sato S, Kuwana M, Aggarwal R. anti-melanoma differentiation-associated gene 5 is associated with rapidly progressive lung disease and poor survival in us patients with amyopathic and myopathic dermatomyositis. Arthritis Care Res (Hoboken). 2016;68:689-94.

12. Hall JC, Casciola-Rosen L, Samedy LA, Werner J, Owoyemi K, Danoff SK, et al. Anti-melanoma differentiation-associated protein 5-associdade dermatomyositis: expanding the clinical spectrum. Arthritis Care Res (Hoboken). 2013;65:1307-15.

13. Cao H, Pan M, Kang Y, Xia Q, Li X, Zhao $X$, et al. Clinical manifestations of dermatomyositis and clinically amyopathic dermatomyositis patients with positive expression of anti-melanoma differentiation-associated gene 5 antibody. Arthritis Care Res (Hoboken). 2012;64:1602-10.
14. Labrador-Horrillo M, Martinez MA, Selva-O'Callaghan A, Trallero-Araguas E, Balada E, Vilardell-Tarres M, et al. Anti-MDA5 antibodies in a large Mediterranean population of adults with dermatomyositis. J Immunol Res. 2014;2014:290797.

15. Ceribelli A, Fredi M, Taraborelli M, Cavazzana I, Tincani A, Selmi C, et al. Prevalence and clinical significance of anti-MDA5 antibodies in European patients with polymyositis/dermatomyositis. Clin Exp Rheumatol. 2014;32:891-7.

16. Sato S, Murakami A, Kuwajima A, Takehara K, Mimori T, Kawakami A, et al. Clinical utility of an enzyme-linked immunosorbent assay for detecting anti-melanoma differentiation-associated gene 5 autoantibodies. PLoS One. 2016;11:e0154285.

17. Medical Research Council. Memorandum No. 45. Aids to the examination of the peripheral nervous system. London: Her Majesty's Stationery Office; 1981.

18. Muro Y, Sugiura K, Hoshino K, Akiyama M. Disappearance of anti-MDA-5 autoantibodies in clinically amyopathic DM/interstitial lung disease during disease remission. Rheumatology (Oxford). 2012;51:800-4.

19. Matsushita T, Mizumaki K, Kano M, Yagi N, Tennichi M, Takeuchi Aetal. Anti-MDA-5 antibody level is a novel tool for monitoring disease activity in rapidly progressive interstitial lung disease with dermatomyositis. Br J Dermatol. 2017;176:395-402.

20. Sato S, Kuwana M, Fujita T, Suzuki Y. Anti-CADM-140/MDA5 autoantibody titer correlates with disease activity and predicts disease outcome in patients with dermatomyositis and rapidly progressive interstitial lung disease. Mod Rheumatol. 2013;23:496-502

21. Mukae H, Ishimoto H, Sakamoto N, Hara S, Kakugawa T, Nakayama S, et al. Clinica differences between interstitial lung disease associated with clinically amyopathic dermatomyositis and classic dermatomyositis. Chest. 2009;136:1341-1347.

22. Ye S, Chen XX, Lu XY, Wu MF, Deng Y, Huang WQ, et al. Adult clinically amyopathic dermatomyositis with rapid progressive interstitial lung disease: a retrospective cohort study. Clin Rheumatol. 2007;26:1647-54.

23. Ji SY, Zeng FQ, Guo Q, Tan GZ, Tang HF, Luo YJ,et al. Predictive factors and unfavorable prognostic factors of interstitial lung disease in patients with polymyositis or dermatomyositis: a retrospective study. Chin Med J (Engl). 2010;123:517-22

24. Sontheimer RD. clinically amyopathic dermatomyositis: what can we now tell our patients? Arch Dermatol. 2010;146:76-80.

25. Sato S, Kuwana M. clinically amyopathic dermatomyositis. Curr Opin Rheumatol. 2010;22:639-43.

\section{AUTHORS CONTRIBUTION}

Isabela Bruna Pires Borges

(iD) ORCID 0000-0002-5207-7603

Approval of the final version of the manuscript; Conception and planning if the study Elaboration and writing of the manuscript; Obtaining, analyzing and interpreting the data; Effective participation in research orientation; Intellectual participation in prodata; Effective participation in research orientation; Intellectual participation in pro-
paedeutic and/or therapeutic conduct of cases studied; Critical review of the literature; Critical review of the manuscrip

Marilda Guimarães Silva iD ORCID 0000-0001-5668-1130

Approval of the final version of the manuscript; Elaboration and writing of the manuscript; Obtaining, analyzing and interpreting the data; Intellectual participation in propaedeutic and/or therapeutic conduct of cases studied; Critical review of the literature; Critical review of the manuscript
Samuel Katsuyuki Shinjo ORCID 0000-0002-3682-4517

Statistical analysis; Approval of the final version of the manuscript; Conception and planning if the study; Elaboration and writing of the manuscript; Obtaining, analyzing and interpreting the data; Effective participation in research orientation; Intellectual participation in propaedeutic and/or therapeutic conduct of cases studied; Critical review of the literature; Critical review of the manuscript

How to cite this article: Borges IBP, Silva MG, Shinjo SK. Prevalence and reactivity of anti-melanoma differentiation-associated gene 5 (antiMDA-5) autoantibody in Brazilian patients with dermatomyositis. An Bras Dermatol. 2018;93(4):517-23. 